\title{
Right-angled Artin groups and enhanced Koszul properties
}

\author{
Alberto Cassella and Claudio Quadrelli* \\ Communicated by Pavel A. Zalesskii
}

\begin{abstract}
Let $\mathbb{F}$ be a finite field. We prove that the cohomology algebra $H^{\bullet}\left(G_{\Gamma}, \mathbb{F}\right)$ with coefficients in $\mathbb{F}$ of a right-angled Artin group $G_{\Gamma}$ is a strongly Koszul algebra for every finite graph $\Gamma$. Moreover, $H^{\bullet}\left(G_{\Gamma}, \mathbb{F}\right)$ is a universally Koszul algebra if, and only if, the graph $\Gamma$ associated to the group $G_{\Gamma}$ has the diagonal property. From this, we obtain several new examples of pro- $p$ groups, for a prime number $p$, whose continuous cochain cohomology algebra with coefficients in the field of $p$ elements is strongly and universally (or strongly and non-universally) Koszul. This provides new support to a conjecture on Galois cohomology of maximal pro- $p$ Galois groups of fields formulated by J. Mináč et al.
\end{abstract}

\section{Introduction}

Right-angled Artin groups - RAAGs for short - are a combinatorial construction that has played a prominent role in geometric group theory in recent decades. An RAAG is defined by a presentation where all relations are commutators of weight 2 of the generators, which comes equipped with a combinatorial graph whose vertices are the generators, and two vertices are joined by an edge whenever they commute. RAAGs may seem the most elementary class among Artin groups, yet such groups have surprising richness and flexibility, and this led to some remarkable applications. (For an overview on RAAGs, we refer to [1].)

In the present paper, we investigate enhanced Koszul properties for the cohomology of finitely generated RAAGs. It is well known that the cohomology algebra $H^{\bullet}\left(G_{\Gamma}, \mathbb{F}\right)=\bigoplus_{n \geq 0} H^{n}\left(G_{\Gamma}, \mathbb{F}\right)$ of an RAAG $G_{\Gamma}$ with associated graph $\Gamma$, with coefficients in a finite field $\mathbb{F}$ (considered as trivial $G_{\Gamma}$-module) and endowed with the graded-commutative cup-product

$$
H^{r}\left(G_{\Gamma}, \mathbb{F}\right) \otimes_{\mathbb{F}} H^{s}\left(G_{\Gamma}, \mathbb{F}\right) \stackrel{\cup}{\longrightarrow} H^{r+s}\left(G_{\Gamma}, \mathbb{F}\right), \quad r, s \geq 0
$$

is isomorphic to the exterior Stanley-Reisner algebra induced by $\Gamma$, and thus it is a quadratic algebra, i.e., a graded algebra which is generated by elements of degree 1 , and with homogeneous defining relations of degree 2 . By a result of 
R. Fröberg, the algebra $H^{\bullet}\left(G_{\Gamma}, \mathbb{F}\right)$ is also Koszul (cf. [12] and [20]). The Koszul property for quadratic algebras was singled out by S. Priddy in [27], and yields exceptionally nice behavior in terms of cohomology (cf. Definition 2.1 below and [22, Chapter 2]). The Koszul property is very restrictive, still it arises in various areas of mathematics, such as representation theory, algebraic geometry, combinatorics and Galois theory.

Recently, some stronger versions of the Koszul property were introduced and investigated in commutative algebra (see, e.g., $[3,4,11,13]$ ) and extended to the noncommutative setting (cf. [21]), and finally considered in the context of Galois cohomology (cf. [17,30]). In particular, one has the notion of strongly Koszul algebra and universally Koszul algebra. These two "enhanced versions" of Koszulity are independent to each other and imply the "simple" Koszulity (see Subsection 2.2 and $[17, \S 2])$. Usually, checking whether a given quadratic algebra is Koszul is a rather hard problem. Surprisingly, testing these enhanced versions of the Koszul property may be easier, even though they are more restrictive.

For RAAGs we prove the following.

Theorem 1.1. Let $\Gamma$ be a finite combinatorial graph and $G_{\Gamma}$ the associated $R A A G$, and let $\mathbb{F}$ be a finite field. The cohomology algebra $H^{\bullet}\left(G_{\Gamma}, \mathbb{F}\right)$ is strongly Koszul.

RAAGs of elementary type are the RAAGs which are constructible starting from free abelian groups and taking direct products with $\mathbb{Z}$ and free products (see Definition 3.5). Equivalently, $G_{\Gamma}$ is of elementary type if $\Gamma$ has the diagonal property i.e., $\Gamma$ does not contain squares or length-3 paths as full subgraphs (see [39] and Proposition 3.6). For example, if $\Gamma$ is complete or a star, then $G_{\Gamma}$ is of elementary type. This property characterizes those RAAGs whose cohomology is universally Koszul.

Theorem 1.2. Let $\Gamma$ be a finite combinatorial graph and $G_{\Gamma}$ the associated $R A A G$, and let $\mathbb{F}$ be a finite field. The cohomology algebra $H^{\bullet}\left(G_{\Gamma}, \mathbb{F}\right)$ is universally Koszul if, and only if, $G_{\Gamma}$ is of elementary type.

Interestingly, Theorems 1.1 and 1.2 provide plenty of examples of strongly Koszul algebras which are not universally Koszul.

For a prime number $p$ and a graph $\Gamma$, let $\varepsilon_{\Gamma}$ denote the pro- $p$ completion of the RAAG $G_{\Gamma}$, and let $\mathbb{F}_{p}$ denote the field with $p$ elements. A result of $\mathrm{K}$. Lorensen states that the (continuous cochain) $\mathbb{F}_{p}$-cohomology algebra of $\mathscr{E}_{\Gamma}$ coincides with the algebra $H^{\bullet}\left(G_{\Gamma}, \mathbb{F}_{p}\right)$ (cf. [16]). Thus, Theorems 1.1 and 1.2 yield several new examples of pro- $p$ groups with $\mathbb{F}_{p}$-cohomology which is strongly and universally (or non-universally) Koszul, in particular among generalized pro- $p$ RAAG, a class of pro- $p$ groups introduced in [31] (see Subsection 5.1). 
This has great relevance in the context of Galois theory. Let $\mathbb{K}$ be a field containing a root of 1 of order $p$, and let $\mathscr{E}_{\mathbb{K}}$ denote the maximal pro- $p$ Galois group of $\mathbb{K}$ - i.e., $\boldsymbol{E}_{\mathbb{K}}$ is the Galois group of the maximal pro- $p$-extension of $\mathbb{K}$. In the last two decades, Koszulity has gained importance in Galois cohomology, thanks to the work of L. Positselski, especially in connection with the celebrated BlochKato conjecture (see, e.g., [23, 25, 26]). In particular, Positselski conjectured that the $\mathbb{F}_{p}$-cohomology algebra of a maximal pro- $p$ Galois group $\mathscr{E}_{\mathbb{K}}$ is Koszul, and this was shown to be true in some relevant cases (cf. [18, 24, 29]). More recently, in [17], J. Mináč et al. conjectured that the $\mathbb{F}_{p}$-cohomology algebra of a maximal pro- $p$ Galois group $\mathscr{E}_{\mathbb{K}}$ is universally Koszul, and proved this in some cases.

Conjecture 1.3 ([17, Conjecture 2]). Let $\mathbb{K}$ be a field containing a root of 1 of order $p$, and suppose that $\mathscr{E}_{\mathbb{K}}$ is finitely generated. Then the $\mathbb{F}_{p}$-cohomology algebra of $\mathcal{E}_{\mathbb{K}}$ is universally Koszul.

If $\Gamma$ is a graph with the diagonal property, then it is well known that the pro- $p$ RAAG $\mathscr{E}_{\Gamma}$ associated to $\Gamma$ occurs as the maximal pro- $p$ Galois group $\mathscr{E}_{\mathbb{K}}$ for some field $\mathbb{K}$ containing a root of 1 of order $p$ (see Proposition 5.8 below). On the other hand, it was recently shown that if $\Gamma$ is a graph without the diagonal property, then it cannot occur as the maximal pro- $p$ Galois group $\mathscr{E}_{\mathbb{K}}$ for any field $\mathbb{K}$ containing a root of 1 of order $p$ (see [28, Theorem 5.6] and [34, Theorem 1.2]). Therefore, from the pro- $p$ version of Theorem 1.2, one deduces the following Galois-theoretic result.

Corollary 1.4. A pro- $p$ RAAG $\mathscr{G}_{\Gamma}$ has universally Koszul $\mathbb{F}_{p}$-cohomology if, and only if, there exists a field $\mathbb{K}$ containing a root of 1 of order $p$ such that $\mathscr{E}_{\Gamma} \simeq \mathscr{E}_{\mathbb{K}}$.

This settles Conjecture 1.3 positively for the class of maximal pro- $p$ Galois groups of fields which are pro- $p$ RAAGs.

\section{Quadratic algebras and Koszul properties}

A graded algebra over a field $\mathbb{F}$ is an associative algebra $A_{\bullet}$ which decomposes as a direct sum of vector spaces $\bigoplus_{n \in \mathbb{Z}} A_{n}$ such that $A_{n} \cdot A_{m} \subseteq A_{m+n}$. Hereinafter, every graded algebra $A_{\bullet}$ is assumed to satisfy the following conditions: $A_{n}=0$ for $n<0, A_{1}=\mathbb{F}, \operatorname{dim}\left(A_{n}\right)<\infty$, and $\mathbb{F}$ is always assumed to be a finite field.

For a subset $S$ of $A_{\bullet},(S)$ denotes the two-sided ideal of $A_{\bullet}$ generated by $S$. Moreover, $A_{+}$denotes the augmentation ideal $\bigoplus_{n \geq 1} A_{n}$ of $A_{\bullet}$. Finally, if $S$ is a subset of a vector space $V$, then $\langle S\rangle$ denotes the vector subspace generated by $S$. 


\subsection{Quadratic algebras and Koszul algebras}

For a vector space $V$, let $T_{\bullet}(V)=\bigoplus_{n \geq 0} V^{\otimes n}$ denote the tensor algebra generated by $V$. A graded algebra $A$. is called quadratic if there exists an isomorphism of graded algebras $A_{\bullet} \simeq T_{\bullet}(V) /(\Omega)$ for some vector space $V$ and some subset $\Omega \subseteq V \otimes V$. We write $T_{\bullet}(V) /(\Omega)=Q(V, \Omega)$.

An ideal $I \unlhd A_{\bullet}$ inherits the grading from $A_{\bullet}$, i.e.,

$$
I=\bigoplus_{n \geq 1} I_{n}, \quad I_{n}=A_{n} \cap I .
$$

In particular, $A_{+}=\bigoplus_{n \geq 1} A_{n}$ is the augmentation ideal of $A_{\bullet}$.

Definition 2.1. A quadratic algebra $A_{\bullet}$ is said to be Koszul if it admits a resolution

$$
\cdots \rightarrow P(2) \bullet \rightarrow P(1) \bullet \rightarrow P(0) \bullet \rightarrow \mathbb{F}
$$

of right $A_{\bullet}$-modules (with trivial action on $\mathbb{F}$ ), where, for each $i \geq 0$, we have that $P(i)_{\bullet}=\bigoplus_{n \geq 0} P(i)_{n}$ is a free graded $A_{\bullet}$-module such that $P(n)_{n}$ is finitely generated for all $n \geq 0$.

We will not need the formal definition of Koszul algebra for our investigation. For further properties of Koszul algebras, we direct the reader to [22, Chapter 2] and to $[18, \S 2]$.

Example 2.2. Suppose that $V$ is a finite-dimensional vector space. The tensor algebra $T_{\bullet}(V)$, the exterior algebra $\Lambda_{\bullet}(V)$ and the quadratic algebra $Q\left(V, V^{\otimes 2}\right)$ (called the trivial quadratic algebra) are Koszul (cf. [15, Example 3.2.5]).

Given two quadratic algebras $A_{\bullet}=Q\left(A_{1}, \Omega_{A}\right)$ and $B_{\bullet}=Q\left(B_{1}, \Omega_{B}\right)$, one has the following constructions (cf. [18, Example 2.5]).

(a) The direct product of $A_{\bullet}$ and $B_{\bullet}$ is the quadratic algebra

$$
A_{\bullet} \sqcap B_{\bullet}=Q\left(A_{1} \oplus B_{1}, \Omega\right),
$$

with

$$
\Omega=\left\langle\Omega_{A} \cup \Omega_{B} \cup\left(A_{1} \otimes B_{1}\right) \cup\left(B_{1} \otimes A_{1}\right)\right\rangle .
$$

(b) The wedge product (or skew-symmetric tensor product) of $A_{\bullet}$ and $B_{\bullet}$ is the quadratic algebra $A_{\bullet} \wedge B_{\bullet}=Q\left(A_{1} \oplus B_{1}, \Omega\right)$, with $\Omega=\left\langle\Omega_{A} \cup \Omega_{B} \cup \Omega_{\wedge}\right\rangle$, where

$$
\Omega_{\wedge}=\left\langle a b+b a, a \in A_{1}, b \in B_{1}\right\rangle \subseteq A_{1} \otimes B_{1} \oplus B_{1} \otimes A_{1} .
$$

If both $A_{\bullet}$ and $B_{\bullet}$ are Koszul, then also their direct product and wedge product are Koszul (cf. [22, § 3.1]). 


\subsection{Enhanced Koszul properties}

Let $A_{\bullet}$ be a graded algebra. For two ideals $I, J$ of $A_{\bullet}$, the colon ideal $I: J$ is the ideal

$$
I: J=\left\{a \in A_{\bullet} \mid a \cdot J \subseteq I\right\} .
$$

In particular, if $I=(0)$, then one has

$$
\text { (0) }: J=\operatorname{Ann}(J)=\{a \in A \bullet \mid a \cdot J=0\} .
$$

Note that, for all ideals $I, J$ of $A_{\bullet}$, one has $I \subseteq I: J$ and $\operatorname{Ann}(J) \subseteq I: J$.

Following [17, § 2.2], we state the definitions of the following three "enhanced versions" of the Koszul property: strong Koszulity, universal Koszulity and the PBW property.

Definition 2.3 ([17, Definition 12]). A quadratic algebra $A_{\bullet}$ is said to be strongly Koszul if $A_{1}$ has a basis

$$
\mathcal{X}=\left\{u_{1}, \ldots, u_{d}\right\}
$$

such that, for every subset $\mathcal{Y}=\left\{u_{i_{1}}, \ldots, u_{i_{k}}\right\}$ of $\mathcal{X}$ and for every $r \in\{1, \ldots, k-1\}$, the colon ideal $\left(u_{i_{1}}, \ldots, u_{i_{r-1}}\right):\left(u_{i_{r}}\right)$ is generated by a subset of $\mathcal{X}$.

(See $[4,13]$ for the original definition of the strong Koszulity property in commutative algebra.)

For a quadratic algebra $A_{\bullet}$, let

$$
\mathscr{L}\left(A_{\bullet}\right)=\left\{I \unlhd A_{\bullet} \mid I=A_{\bullet} \cdot I_{1}\right\}
$$

denote the set of all ideals of $A_{\bullet}$ generated by a subset of $A_{1}$. In particular, both the trivial ideal (0) and the augmentation ideal $A_{+}$belong to $\mathscr{L}\left(A_{\bullet}\right)$.

Definition 2.4 (cf. [17, Proposition 17]). A quadratic algebra $A \bullet$ is said to be universally Koszul if, for every ideal $I \in \mathscr{L}\left(A_{\bullet}\right)$ and every $b \in A_{1} \backslash I_{1}$, one has $I:(b) \in \mathscr{L}\left(A_{\bullet}\right)$.

(See $[3,5]$ for the original definition of the universal Koszulity property in commutative algebra.)

Examples 2.5. Consider the following examples.

(a) Set $A_{\bullet}=\mathbb{F}[a]$, i.e., $A_{\bullet}$ is the free graded algebra on the generator $a$. The augmentation ideal $A_{+}$is the ideal $(a)$, and one has $\mathscr{L}\left(A_{\bullet}\right)=\{(0),(a)\}$. Then $(0):(a)=\operatorname{Ann}(a)$, and therefore $(0):(a)=(0)$, which lies in $\mathscr{L}\left(A_{\bullet}\right)$. Hence, $A \bullet$ is both strongly and universally Koszul. 
(b) Set $A_{\bullet}=\mathbb{F}[a] /\left(a^{2}\right)$, i.e., $A_{\bullet}$ is the algebra generated by $a$ and concentrated in degree 0 and 1 . One has $A_{+}=A_{1}=(a)$, and $\mathscr{L}=\{(0),(a)\}$. Moreover, $(0):(a)=\operatorname{Ann}(a)=A_{1} \in \mathscr{L}\left(A_{\bullet}\right)$. Hence, $A_{\bullet}$ is both strongly and universally Koszul.

One has the following two properties for universally Koszul algebras.

Proposition 2.6. Let $A_{\bullet}$ and $B_{\bullet}$ be quadratic algebras. Then the direct product $A_{\bullet} \sqcap B_{\bullet}$ is universally Koszul if, and only if, both $A_{\bullet}$ and $B_{\bullet}$ are universally Koszul.

Proof. Let us assume that $A_{\bullet}$ is not universally Koszul. Then there exists an ideal $I$ of $A_{\bullet}, I \in \mathscr{L}\left(A_{\bullet}\right)$, and an element $\beta \in A_{1}$ such that $J \notin \mathscr{L}\left(A_{\bullet}\right)$, for $J$ the colon ideal $I:(b)$.

Set $C_{\bullet}=A_{\bullet} \sqcap B_{\bullet}$, and let $\tilde{I}$ be the extension of $I$ in $C_{\bullet}$. Then $\tilde{I}=I$, as $A_{+} \cdot B_{+}=0$. Let $\tilde{J}$ denote the colon ideal $\tilde{I}:(b) \unlhd C_{\bullet}$. Then $\tilde{J}=J \oplus B_{+}$, and there exists an element $c \in J_{+} \subseteq \tilde{J}_{+}$such that $c \notin\left(J_{1}\right)$, and thus

$$
c \notin\left(\tilde{J}_{1}\right)=\left(J_{1}+B_{1}\right) .
$$

The opposite implication is [17, Proposition 28].

Proposition 2.7. [17, Proposition 31] Let A• be a quadratic universally Koszul algebra, and let $V$ be a vector space of finite dimension. Then the wedge product $A_{\bullet} \wedge \Lambda_{\bullet}(V)$ is universally Koszul.

Finally, one has also the notion of $P B W$ generators of a quadratic algebra introduced in [22, Chapter 4] - which generalizes the notion of G-quadratic commutative algebra (cf. [2]). A quadratic algebra $A_{\bullet}=Q(V, \Omega)$ is called a $P B W$ quadratic algebra if it admits generators for which the non-commutative Gröbner basis of relations consists of elements of degree two (see [15, §4.3] and [17, Definition 8]). Namely, consider the lexicographical order $\prec$ on the set of multi-indices of length $n$-i.e., $\left(i_{1}, \ldots, i_{n}\right) \prec\left(j_{1}, \ldots, j_{n}\right)$ if, and only if, there exists $1 \leq k \leq n$ such that $i_{1}=j_{1}, i_{2}=j_{2}, \ldots, i_{k-1}=j_{k-1}$ and $i_{k}<j_{k}$ for $i_{h}, j_{h} \in\{1, \ldots, d\}$, where $d=\operatorname{dim}(V)$.

Let $\left\{v_{1}, \ldots, v_{d}\right\}$ be a basis of $V$. Then there exists $\delta \subseteq\{1, \ldots, d\}^{2}$ such that the relations in $\Omega$ can be written in the form

$$
v_{i_{1}} v_{i_{2}}=\sum_{\substack{\left(j_{1}, j_{2}\right) \prec\left(i_{1}, i_{2}\right) \\\left(j_{1}, j_{2}\right) \in \mathcal{S}}} \alpha v_{j_{1}} v_{j_{2}}, \quad\left(i_{1}, i_{2}\right) \notin \delta, \alpha \in \mathbb{F}
$$

(cf. [22, Lemma 4.1.1]). 
Definition 2.8. Given $A \bullet$ and $\delta$ as above, set $\delta^{(0)}=\{\varnothing\}, \delta^{(1)}=\{1, \ldots, d\}$ and

$$
8^{(n)}=\left\{\left(i_{1}, \ldots, i_{n}\right) \mid\left(i_{h}, i_{h+1}\right) \in \delta, h=1, \ldots, n-1\right\} \text { for } n \geq 2 .
$$

The elements $v_{1}, \ldots, v_{d}$ of a basis of $V$ are called $P B W$ generators of $A \bullet$ if the set of monomials $\left\{v_{i_{1}} \cdots v_{i_{n}} \mid\left(i_{1}, \ldots, i_{n}\right) \in 8^{(n)}\right\}$ is a basis of $A_{n}$ for every $n \geq 0$. Such a quadratic algebra is called a PBW algebra.

These "enhanced Koszulity" properties are independent of each other, i.e., none implies any other. On the other hand, if a quadratic algebra has one of these properties, then it is Koszul. Altogether, one has the following picture (cf. [17, § 1.2]):

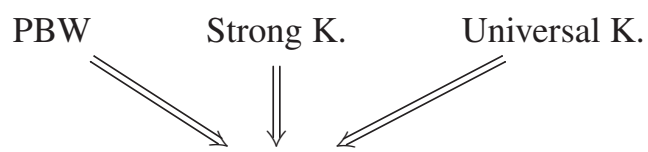

Koszulity.

\section{Right-angled Artin groups}

\subsection{Graphs}

For the notion of graph, see [6, Chapter 1]. A naïve graph is a pair $\Gamma=(\mathcal{V}, \mathcal{E})$ of sets, where $\mathcal{E} \subseteq[\mathcal{V}]^{2}$, i.e., the elements of $\mathcal{E}$ are unordered subsets of 2 elements of $\mathcal{V}$, which we shall denote by $(v, w)=(w, v)$, with $v, w \in \mathcal{V}$. The elements of $\mathcal{V}$ are the vertices of the graph $\Gamma$; the elements of $\mathcal{E}$ are its edges. Moreover, we assume all graphs to have no loops, i.e., $(v, v) \notin \mathcal{E}$ for any $v \in \mathcal{V}$. A graph $\Gamma=(\mathcal{V}, \mathscr{E})$ is said to be finite if it has finite vertices.

Henceforth, every graph will be assumed to be naïve and finite. Here we list some definitions regarding graphs which will be used hereinafter.

Definition 3.1. Let $\Gamma=(\mathcal{V}, \mathcal{E})$ be a graph.

(i) $\Gamma$ is a complete graph if $\mathcal{E}=[\mathcal{V}]^{2}$.

(ii) A star graph is a graph $\Gamma=(\mathcal{V}, \mathcal{E})$ such that $\mathcal{V}=\left\{w, v_{1}, \ldots, v_{d}\right\}$, with $d \geq 2$, and $\mathcal{E}=\left\{\left(w, v_{1}\right), \ldots,\left(w, v_{d}\right)\right\}$.

(iii) A full subgraph (or induced subgraph) of $\Gamma$ is a subgraph $\Gamma^{\prime}=\left(\mathcal{V}^{\prime}, \mathcal{E}^{\prime}\right)$ of $\Gamma$ such that $\mathcal{E}^{\prime}=\mathscr{E} \cap\left[\mathcal{V}^{\prime}\right]^{2}$, i.e., if two vertices of $\mathcal{V}^{\prime}$ are joined by an edge of $\Gamma$, then they are joined by an edge also in $\Gamma^{\prime}$.

(iv) For $n \geq 1$, an $n$-clique $\Gamma^{\prime}$ of $\Gamma$ is a full subgraph $\Gamma^{\prime}$ of $\Gamma$ with $n$ vertices which is a complete graph. 
(v) For $v, w \in \mathcal{V}$, a path from $v$ to $w$ is a subgraph $P=\left(\mathcal{V}^{\prime}, \mathcal{E}^{\prime}\right)$ of $\Gamma$ with

$$
\begin{aligned}
& \mathcal{V}^{\prime}=\left\{v_{0}=v, v_{1}, \ldots, v_{n-1}, v_{n}=w\right\}, \\
& \mathcal{E}^{\prime}=\left\{\left(v_{0}, v_{1}\right),\left(v_{1}, v_{2}\right), \ldots,\left(v_{n-1}, v_{n}\right)\right\},
\end{aligned}
$$

and $n$ is the length of $P$. A path is a cycle if $v=w$.

\subsection{RAAGs and cohomology}

Let $\Gamma=(\mathcal{V}, \mathcal{E})$ be a graph, with vertices $\mathcal{V}=\left\{v_{1}, \ldots, v_{d}\right\}$. The right-angled Artin group associated to $\Gamma$ is the group $G_{\Gamma}$ with presentation

$$
\left.G_{\Gamma}=\left\langle v_{1}, \ldots, v_{d}\right|\left[v_{i}, v_{j}\right]=1 \text { for }\left(v_{i}, v_{j}\right) \in \mathcal{E}\right\rangle .
$$

The following is a well-known result on RAAGs.

Lemma 3.2. Let $\Gamma$ be a graph, and suppose $\Gamma$ decomposes in connected components $\Gamma_{1}, \ldots, \Gamma_{r}$. Then the $R A A G G_{\Gamma}$ decomposes as free product $G_{\Gamma_{1}} * \cdots * G_{\Gamma_{r}}$.

Definition 3.3. Let $\Gamma=(\mathcal{V}, \mathcal{E})$ be a graph, with $\mathcal{V}=\left\{v_{1}, \ldots, v_{d}\right\}$, and let $V$ be the $\mathbb{F}$-vector space generated by $\mathcal{V}^{\mathrm{op}}=\left\{a_{1}, \ldots, a_{d}\right\}$. The exterior StanleyReisner algebra $\Lambda_{\bullet}\left(\Gamma^{\mathrm{op}}\right)$ over $\mathbb{F}$ associated to $\Gamma$ is the quotient of the exterior algebra $\Lambda_{\bullet}(V)$ over the two-sided ideal generated by

$$
\Omega=\left\langle a_{i} \wedge a_{j} \text { for }\left(v_{i}, v_{j}\right) \notin \mathcal{E}, 1 \leq i, j \leq d\right\rangle \subseteq \Lambda_{2}(V) .
$$

Since $\Lambda_{\bullet}(V)$ is quadratic and $\Omega \subseteq \Lambda_{2}(V)$, the algebra $\Lambda_{\bullet}\left(\Gamma^{\mathrm{op}}\right)$ is quadratic. While working with the algebra $\Lambda_{\bullet}\left(\Gamma^{\mathrm{op}}\right)$, we will omit the wedge product $\wedge$ and write $a b$ for the product of two elements $a$ and $b$ of $\Lambda_{\bullet}\left(\Gamma^{\mathrm{op}}\right)-$ in particular, $a_{i} a_{j}$ will denote the image of $a_{i} \wedge a_{j}$ in $\Lambda_{2}\left(\Gamma^{\mathrm{op}}\right)$. The result of R. Fröberg [12] implies that $\Lambda_{\bullet}\left(\Gamma^{\mathrm{op}}\right)$ is Koszul for any graph $\Gamma$.

The following result describes the $\mathbb{F}$-cohomology algebra of an RAAG (see $[20, \S 3.2]$ and $[38, \S 4.2 .2])$.

Proposition 3.4. Let $G_{\Gamma}$ be the $R A A G$ associated to a graph $\Gamma=(\mathcal{V}, \mathcal{E})$. Then the F-cohomology algebra $H^{\bullet}\left(G_{\Gamma}, \mathbb{F}\right)$ of $G_{\Gamma}$ is isomorphic to the algebra $\Lambda_{\bullet}\left(\Gamma^{\mathrm{op}}\right)$. In particular, $\Lambda_{\bullet}\left(\Gamma^{\mathrm{op}}\right)$ is Koszul.

Thus, if a graph $\Gamma$ decomposes into connected components $\Gamma_{1}, \ldots, \Gamma_{r}$, then the $\mathbb{F}$-cohomology algebra of the RAAG $G_{\Gamma}$ decomposes as direct product of quadratic algebras

$$
H^{\bullet}\left(G_{\Gamma}, \mathbb{F}\right) \simeq \Lambda_{\bullet}\left(\Gamma^{\mathrm{op}}\right) \simeq \Lambda_{\bullet}\left(\Gamma_{1}^{\mathrm{op}}\right) \sqcap \cdots \sqcap \Lambda_{\bullet}\left(\Gamma_{r}^{\mathrm{op}}\right) .
$$




\subsection{RAAGs of elementary type}

By Lemma 3.2, the free product of two RAAGs $G_{\Gamma_{1}} * G_{\Gamma_{2}}$ is the RAAG with the disjoint union of $\Gamma_{1}$ and $\Gamma_{2}$ as associated graph. Analogously, the direct product $G_{\Gamma} \times \mathbb{Z}$ of an RAAG $G_{\Gamma}$ with $\mathbb{Z}$ is isomorphic to the RAAG $G_{\tilde{\Gamma}}$, where $\tilde{\Gamma}$ is the cone graph with basis $\Gamma$, i.e.,

$$
\begin{aligned}
& \mathcal{V}(\tilde{\Gamma})=\mathcal{V}(\Gamma \dot{\cup}\{w\}), \\
& \mathcal{E}(\tilde{\Gamma})=\mathcal{E}(\Gamma) \dot{\cup}\{(w, v), v \in \mathcal{V}(\Gamma)\} .
\end{aligned}
$$

For the following definition, we mimic the definition of elementary type pro- $p$ groups, defined by I. Efrat (cf. [9, §3])

Definition 3.5. The class of RAAGs of elementary type is the minimal class $C$ of finitely generated RAAGs such that

(a) $\mathbb{Z}$ (considered as RAAGs with associated graph a single vertex) belongs to $C$,

(b) if $G_{\Gamma_{1}}$ and $G_{\Gamma_{2}}$ belong to $\ell$, then also $G_{\Gamma_{1}} * G_{\Gamma_{2}}$ belongs to $\ell$,

(c) if $G_{\Gamma}$ belongs to $\ell$, then also $G_{\Gamma} \times \mathbb{Z}$ belongs to $\ell$.

In other words, RAAGs of elementary type are precisely the RAAGs whose associated graphs are constructible starting from the graph with a single vertex, via the following operations: disjoint union of graphs, and cones.

Let $C_{4}$ and $P_{4}$ denote the cycle of length 4 and the path (non-cycle) of length 3 respectively, namely,

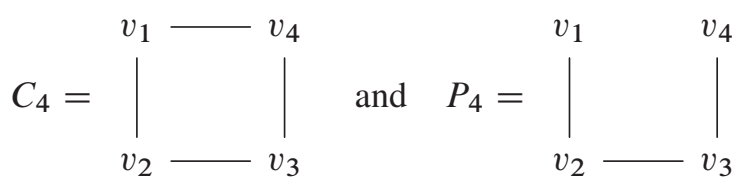

A graph $\Gamma$ is said to have the diagonal property if it does not contain a full subgraph isomorphic to $C_{4}$ or $P_{4}$. By the work of E. S. Wolk [39], one has the following characterization of RAAGs of elementary type.

Proposition 3.6. Let $G_{\Gamma}$ be an RAAG with associated graph $\Gamma$. Then $G_{\Gamma}$ is of elementary type if, and only if, $\Gamma$ has the diagonal property.

Moreover, by [8], every subgroup of an RAAG $G_{\Gamma}$ is again an RAAG if, and only if, $\Gamma$ has the diagonal property. In particular, every subgroup of an RAAG of elementary type is again an RAAG of elementary type. 
Example 3.7. Consider the following examples.

(a) All graphs with at most 3 vertices have the diagonal property and thus yield an RAAG of elementary type.

(b) A star graph $\Gamma$ is the cone graph with basis a disjoint union of vertices, and thus $G_{\Gamma} \simeq(\mathbb{Z} * \cdots * \mathbb{Z}) \times \mathbb{Z}$ is an RAAG of elementary type.

(c) A complete graph $\Gamma$ may be obtained as iterated cone starting from a single vertex; indeed, $G_{\Gamma} \simeq \mathbb{Z} \times \ldots \times \mathbb{Z}$ is an RAAG of elementary type.

\section{RAAGs and enhanced Koszul properties}

Let $\Gamma=(\mathcal{V}, \mathcal{E})$ be a graph, with $\mathcal{V}=\left\{v_{1}, \ldots, v_{d}\right\}$, and set $\mathcal{V}^{\text {op }}=\left\{a_{1}, \ldots, a_{d}\right\}$. Since the product in $\Lambda_{\bullet}\left(\Gamma^{\mathrm{op}}\right)$ is graded-commutative, every ideal in $\Lambda_{\bullet}\left(\Gamma^{\mathrm{op}}\right)$ is two-sided.

Given indices $1 \leq i_{1}<\cdots<i_{n} \leq d$, one has $a_{i_{1}} \cdots a_{i_{n}} \neq 0$ if, and only if, there is an $n$-clique $\Gamma^{\prime}$ of $\Gamma$ such that $\mathcal{V}\left(\Gamma^{\prime}\right)=\left\{v_{i_{1}}, \ldots, v_{i_{n}}\right\}$. In particular, we have $\Lambda_{n}\left(\Gamma^{\mathrm{op}}\right)=0$ if there are no $n$-cliques in $\Gamma-$ which is always the case if $n>d$.

Thus, for every $n \geq 1$, the set

$$
\mathscr{B}_{n}=\left\{a_{i_{1}} \cdots a_{i_{n}} \mid 1 \leq i_{1}<\cdots<i_{n} \leq d \text { and } a_{i_{1}} \cdots a_{i_{n}} \neq 0\right\}
$$

is in 1-to-1 correspondence with the set of all $n$-cliques of $\Gamma$, and it is a basis of $\Lambda_{n}\left(\Gamma^{\mathrm{op}}\right)$. Moreover, for $\Delta=a_{i_{1}} \cdots a_{i_{n}} \in \mathscr{B}_{n}$, we define

$$
\mathcal{V}(\Delta)=\left\{a_{i_{1}}, \ldots, a_{i_{n}}\right\} \subseteq \mathcal{V}^{\text {op }},
$$

namely, $\mathcal{V}(\Delta)$ corresponds to the vertices of the $n$-clique of $\Gamma$ associated to $\Delta$.

Lemma 4.1. For a graph $\Gamma$, let 8 be a subset of $\mathcal{V}^{\mathrm{op}}$, and let $I \unlhd \Lambda_{\bullet}\left(\Gamma^{\mathrm{op}}\right)$ be the ideal generated by 8 . Then $I_{n}$ is the subspace of $\Lambda_{n}\left(\Gamma^{\mathrm{op}}\right)$ generated by

$$
\mathscr{B}(8)_{n}=\left\{\Delta \in \mathscr{B}_{n} \mid \mathcal{V}(\Delta) \cap \S \neq \varnothing\right\} .
$$

Proof. We proceed by induction on $n$. If $n=1$, then $\mathscr{B}(8)_{n}=\&$. If $n \geq 2$, then, by induction, one has

$$
\begin{aligned}
I_{n} & =I_{1} \cdot \Lambda_{n-1}\left(\Gamma^{\mathrm{op}}\right)+\cdots+I_{n-1} \cdot \Lambda_{1}\left(\Gamma^{\mathrm{op}}\right) \\
& =\langle \&\rangle \cdot\left\langle\mathscr{B}_{n-1}\right\rangle+\cdots+\left\langle\mathscr{B}(\&)_{n-1}\right\rangle \cdot\left\langle\mathscr{B}_{1}\right\rangle
\end{aligned}
$$

and thus $I_{n}=\left\langle\mathscr{B}(\delta)_{n}\right\rangle$. 
From the above description of $\Lambda_{\bullet}\left(\Gamma^{\mathrm{op}}\right)$, one deduces easily the following.

Corollary 4.2. Let $\Gamma=(\mathcal{V}, \mathcal{E})$ be a graph. The exterior Stanley-Reisner algebra $A_{\bullet}=\Lambda_{\bullet}\left(\Gamma^{\mathrm{op}}\right)$ is a PBW algebra.

Proof. Set $\AA^{(n)}=\left\{\left(i_{1}, \ldots, i_{n}\right) \mid 1 \leq i_{1}<\cdots<i_{n} \leq d\right\}$ for every $n \geq 1$. Then one may write the relations of $A_{\bullet}$ as $a_{j} a_{i}=\alpha a_{i} a_{j}$, with $i<j$ and $\alpha=-1$ if $\left(v_{i}, v_{j}\right) \in \mathcal{E}$ and $\alpha=0$ otherwise, and moreover $a_{i}^{2}=0$ for all $i$. So, the sets $\mathcal{S}^{(n)}$ are as in Definition 2.8. Thus, the set $\mathcal{V}^{\mathrm{op}}=\left\{a_{1}, \ldots, a_{d}\right\}$ is a set of PBW-generators of $A_{\bullet}$, as $\left\{v_{i_{1}} \cdots v_{i_{n}} \mid\left(i_{1}, \ldots, i_{n}\right) \in \mathcal{\odot}^{(n)}\right\}=\mathscr{B}_{n}-$ which is a basis of $A_{n}-$ for every $n \geq 0$.

\subsection{Strong Koszulity}

The first result we get is the strong Koszulity of $\Lambda_{\bullet}\left(\Gamma^{\mathrm{op}}\right)$, regardless of the graph $\Gamma$.

Theorem 4.3. The exterior Stanley-Reisner algebra $\Lambda_{\bullet}\left(\Gamma^{\mathrm{op}}\right)$ is strongly Koszul for any graph $\Gamma=(\mathcal{V}, \mathcal{E})$.

Proof. Set $A_{\bullet}=\Lambda_{\bullet}\left(\Gamma^{\mathrm{op}}\right)$. Our goal is to show that the basis $\mathcal{V}^{\text {op }}$ is the suitable basis of $A_{1}$ fulfilling the condition as in Definition 2.3.

Fix a subset $\mathcal{Y}=\left\{a_{i_{1}}, \ldots, a_{i_{n}}\right\}$ of $\mathcal{V}^{\text {op }}$. For $1 \leq r \leq n$, set

$$
\begin{aligned}
\mathcal{S}^{\prime} & =\left\{a_{i_{1}}, \ldots, a_{i_{r-1}}\right\} \\
I & =\left(a_{i_{1}}, \ldots, a_{i_{r-1}}\right)=\left(\AA^{\prime}\right), \\
J & =I:\left(a_{i_{r}}\right)=\left\{b \in A \bullet \mid a_{i_{r}} \cdot b \in\left(a_{i_{1}}, \ldots, a_{i_{r-1}}\right)\right\} .
\end{aligned}
$$

Moreover, set $\mathcal{S}^{\prime \prime}=\left\{a_{j} \in \mathcal{V}^{\text {op }} \mid a_{j} a_{i_{r}}=0\right\}$ and

$$
\S=\wp^{\prime} \cup \mathcal{S}^{\prime \prime}=\mathcal{V}^{\mathrm{op}} \backslash\left\{a_{j} \mid j \notin\left\{i_{1}, \ldots, i_{r-1}\right\} \text { and } a_{j} a_{i_{r}} \neq 0\right\} .
$$

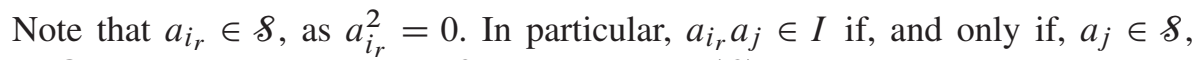
as $\mathcal{B}_{2}$ is a basis of $A_{2}$. Hence, $\delta \subseteq J_{1}$, and $I \subseteq(8) \subseteq J$. We claim that the ideals $(8)$ and $J$ coincide.

Let $b \in A$ • be such that $b \notin(8)$. Thus, one may write

$$
b=\alpha_{1} \Delta_{1}+\cdots+\alpha_{m} \Delta_{m}, \quad \alpha_{h} \in \mathbb{F}^{\times},
$$

where $\Delta_{h} \in \mathscr{B}_{n_{h}}$ for each $h \in\{1, \ldots, m\}$. By Lemma 4.1, $\Delta_{h} \in(\&)$ - respectively $\Delta \in\left(\delta^{\prime}\right)=I, \Delta \in\left(\mathcal{(}^{\prime \prime}\right)$ - if, and only if, the intersection of $\mathcal{V}(\Delta)$ with $\delta$ - respectively with $\mathcal{S}^{\prime}$ and with $\mathcal{S}^{\prime \prime}-$ is not empty. Since $b \notin(\&)$, one has $\Delta_{h} \notin \mathscr{B}(\mathscr{S})_{n_{h}}$ 
for some $h$ in (4.1), and in this case, $a_{i_{r}} \cdot \Delta_{h} \neq 0$. Therefore, one obtains

$$
\begin{aligned}
a_{i_{r}} b & =a_{i_{r}} \cdot \sum_{\Delta_{h} \in(8)} \alpha_{h} \Delta_{h}+a_{i_{r}} \cdot \sum_{\Delta_{h} \notin(8)} \alpha_{h} \Delta_{h} \\
& =\sum_{\Delta_{h} \in I} \alpha_{h} \cdot a_{i_{r}} \Delta_{h}+\sum_{\Delta_{h} \notin(\delta)} \alpha_{h} \cdot a_{i_{r}} \Delta_{h},
\end{aligned}
$$

where $a_{i_{r}} \Delta_{h} \in \mathscr{B}_{n_{h}+1} \backslash \mathcal{B}(\&)_{n_{h}+1}$ and $a_{i_{r}} \Delta_{h} \neq a_{i_{r}} \Delta_{h^{\prime}}$ for every $\Delta_{h}, \Delta_{h^{\prime}} \notin(8)$, $h^{\prime} \neq h$. (Note that if $\Delta_{h} \in \mathscr{B}\left(\mathscr{S}^{\prime \prime}\right)_{n_{h}}$, then $a_{i_{r}} \Delta_{h}=0$.) Consequently, the rightside summand of (4.2) is not trivial, and by Lemma 4.1, it does not lie in $I$, whereas the left-side summand lies in $I$, so that $a_{i_{r}} b \notin I$. Therefore, $b \notin J$, and this proves the inclusion $J \subseteq(8)$.

Theorem 1.1 follows from Theorem 4.3 together with Proposition 3.4.

Remark 4.4. Since strong Koszulity implies Koszulity, Theorem 4.3 provides a new proof of the fact that the algebra $\Lambda_{\bullet}\left(\Gamma^{\mathrm{op}}\right)$ is Koszul.

\subsection{Universal Koszulity}

The following is a direct consequence of Proposition 2.6 and of (3.1).

Proposition 4.5. Let $\Gamma$ be the disjoint union of two graphs $\Gamma_{1}$ and $\Gamma_{2}$. Then $\Lambda_{\bullet}\left(\Gamma^{\mathrm{op}}\right)$ is universally Koszul if, and only if, both $\Lambda_{\bullet}\left(\Gamma_{1}^{\mathrm{op}}\right)$ and $\Lambda_{\bullet}\left(\Gamma_{2}^{\mathrm{op}}\right)$ are universally Koszul.

Theorem 4.6. Let $\Gamma=(\mathcal{V}, \mathcal{E})$ be a graph. The exterior Stanley-Reisner algebra $\Lambda_{\bullet}\left(\Gamma^{\mathrm{op}}\right)$ is universally Koszul if, and only if, $\Gamma$ has the diagonal property.

Proof. Set $A_{\bullet}=\Lambda_{\bullet}\left(\Gamma^{\mathrm{op}}\right)$ and $\mathcal{V}=\left\{v_{1}, \ldots, v_{d}\right\}$

Suppose first that $\Gamma$ has the diagonal property. We proceed by induction on $d$. If $d=1$, then $A_{\bullet} \simeq \mathbb{F}[a] /\left(a^{2}\right)$, which is universally Koszul. If $d=2$, then either $A$ • is the exterior algebra generated by $V$, or it is the trivial algebra generated by $V$, with $V$ a space of dimension 2, so that it is universally Koszul.

If $d \geq 3$, then either $\Gamma$ decomposes as disjoint union of two proper full subgraphs $\bar{\Gamma}_{1}$ and $\Gamma_{2}$, or it is the cone graph with basis a full subgraph $\tilde{\Gamma}$. In the former case, both $\Lambda_{\bullet}\left(\Gamma_{1}^{\mathrm{op}}\right)$ and $\Lambda_{\bullet}\left(\Gamma_{2}^{\mathrm{op}}\right)$ are universally Koszul by induction so that also

$$
A_{\bullet} \simeq \Lambda_{\bullet}\left(\Gamma_{1}^{\mathrm{op}}\right) \sqcap \Lambda_{\bullet}\left(\Gamma_{2}^{\mathrm{op}}\right)
$$

is universally Koszul by Proposition 4.5; in the latter case, one has

$$
A_{\bullet} \simeq \Lambda_{\bullet}\left(\tilde{\Gamma}^{\mathrm{op}}\right) \wedge B_{\bullet}, \quad B_{\bullet} \simeq \mathbb{F}[b] /\left(b^{2}\right),
$$


and $\Lambda_{\bullet}\left(\tilde{\Gamma}^{\text {op }}\right)$ is universally Koszul by induction so that also $A_{\bullet}$ is universally Koszul by Proposition 2.7.

Suppose now that $\Gamma$ does not have the diagonal property. Thus, $\Gamma$ contains a full subgraph $\Gamma^{\prime}$ isomorphic to $C_{4}$ or $P_{4}$. Set $\mathcal{V}=\left\{v_{1}, \ldots, v_{d}\right\}$, with $d \geq 4$, such that $\mathcal{V}\left(\Gamma^{\prime}\right)=\left\{v_{1}, v_{2}, v_{3}, v_{4}\right\}$, with the vertices labelled as in (3.2). Also, set $\mathcal{V}^{\text {op }}=\left\{a_{1}, \ldots, a_{d}\right\}$ and $A_{\bullet}=\Lambda_{\bullet}\left(\Gamma^{\mathrm{op}}\right)$.

Set $b=a_{1}+a_{4}$, and set $J=(0):(b)=\operatorname{Ann}(b)$. Then

$$
b \cdot a_{2} a_{3}=a_{1} a_{2} a_{3}+a_{4} a_{2} a_{3}=0
$$

and $a_{2} a_{3} \in J_{2}$. We claim that $a_{2} a_{3} \notin J_{1} \cdot A_{1}$, i.e., $a_{2} a_{3}$ does not lie in the ideal generated by $J_{1}$, so that $J \notin \mathscr{L}\left(A_{\bullet}\right)$. Clearly, $a_{2}, a_{3} \notin J_{1}$, as $b a_{2}=a_{1} a_{2} \neq 0$ and $a_{3} b=a_{3} a_{4} \neq 0$. Suppose there exist $c_{1}, \ldots, c_{r} \in J_{1}$ and $c_{1}^{\prime}, \ldots, c_{r}^{\prime} \in A_{1}$ such that $c_{1} c_{1}^{\prime}+\cdots+c_{r} c_{r}^{\prime}=a_{2} a_{3}$, and write

$$
c_{h}=\alpha_{1, h} a_{1}+\cdots+\alpha_{d, h} a_{d} \quad \text { and } \quad c_{h}^{\prime}=\beta_{1, h} a_{1}+\cdots+\beta_{d, h} a_{d}
$$

for each $h \in\{1, \ldots, r\}$, with $\alpha_{i, h}, \beta_{i, h} \in \mathbb{F}$. Since $c_{h} \in \operatorname{Ann}(b)$ for every $h$, one has $\alpha_{2, h}=\alpha_{3, h}=0$; otherwise $b c_{h}=\alpha_{2, h} a_{1} a_{2}-\alpha_{3, h} a_{3} a_{4}+\Delta$, with $\Delta \in A_{2}$ a combination of elements $a_{i} a_{j}$ with $i<j,(1,2),(3,4) \neq(i, j)$, and $b c_{h} \neq 0$ as $\mathscr{B}_{2}=\left\{a_{i} a_{j} \mid 1 \leq i<j \leq d, a_{i} a_{j} \neq 0\right\}$ is a basis of $A_{2}$. Thus, one obtains

$$
\begin{aligned}
\sum_{h=1}^{r} c_{h} c_{h}^{\prime} & =\sum_{i<j} a_{i} a_{j}\left(\sum_{h=1}^{r} \alpha_{i, h} \beta_{j, h}-\sum_{h=1}^{r} \alpha_{j, h} \beta_{i, h}\right) \\
& =a_{2} a_{3}\left(\sum_{h=1}^{r} \alpha_{2, h} \beta_{3, h}-\sum_{h=1}^{r} \alpha_{3, h} \beta_{2, h}\right)+\Delta=0+\Delta,
\end{aligned}
$$

with $\Delta \in A_{2}$ a combination of elements $a_{i} a_{j},(i, j) \neq(2,3)$, a contradiction.

Therefore, $a_{2} a_{3} \in J_{2} \backslash J_{1} \cdot A_{1}$, and consequently $J \notin \mathscr{L}\left(A_{\bullet}\right)$, and $A_{\bullet}$ is not universally Koszul.

Theorem 1.2 follows from Theorem 4.6, together with Proposition 3.4, equation (3.1) and Proposition 4.5.

In the following two examples, we show explicitly that $\Lambda_{\bullet}\left(\Gamma^{\mathrm{op}}\right)$ is universally Koszul for two graphs with the diagonal property, without using Proposition 2.7.

Example 4.7. Let $\Gamma=(\mathcal{V}, \mathcal{E})$ be a star graph with

$$
\mathcal{V}=\left\{w, v_{1}, \ldots, v_{d}\right\} \quad \text { and } \quad \mathcal{E}=\left\{\left(w, v_{1}\right), \ldots,\left(w, v_{d}\right)\right\}
$$




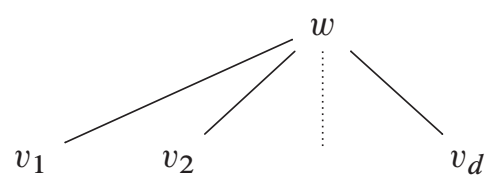

Set $\mathcal{V}^{\text {op }}=\left\{a_{w}, a_{1}, \ldots, a_{d}\right\}$ and $A_{\bullet}=\Lambda_{\bullet}\left(\Gamma^{\mathrm{op}}\right)$. Then

$$
\begin{aligned}
& A_{1}=\left\langle a_{w}, a_{1}, \ldots, a_{d}\right\rangle, \\
& A_{2}=\left\langle a_{w} a_{1}, \ldots, a_{w} a_{d}\right\rangle=a_{w} \wedge A_{1}, \\
& A_{n}=0 \quad \text { for } n \geq 3 .
\end{aligned}
$$

In particular, $a_{i} a_{j}=0$ for all $i, j$. For $I \in \mathscr{L}\left(A_{\bullet}\right)$ and $b \in A_{1} \backslash I_{1}$, write

$$
b=\alpha_{w} a_{w}+\sum_{i} \alpha_{i} a_{i}, \quad \text { with } \alpha_{w}, \alpha_{i} \in \mathbb{F} .
$$

Set $J=I:(b)$. Then we have $b \in J$, and moreover $J_{2}=A_{2}$, as $A_{2} \cdot b \subseteq A_{3}=0$. If $\alpha_{w} \neq 0$, then $A_{1} \cdot b=A_{1} \cdot \alpha_{w} a_{w}=A_{2}=J_{2}$ so that $J$ is 1 -generated, i.e., $J \in \mathscr{L}\left(A_{\bullet}\right)$. If $\alpha_{w}=0$, then $a_{i} b=0$ for all $i \in\{1, \ldots, d\}$, and thus $a_{i} \in J_{1}$. In this case, $a_{w} a_{i} \in A_{1} \cdot J_{1} \subseteq J_{2}$ for all $i$, and therefore $A_{1} \cdot J_{1}=A_{2}$ so that $J$ is 1 -generated, i.e., $J \in \mathscr{L}\left(A_{\bullet}\right)$. Thus, $A_{\bullet}$ is universally Koszul.

Example 4.8. Let $\Gamma$ be the graph

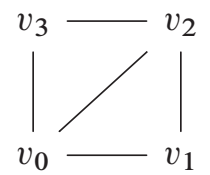

The graph $\Gamma$ is the cone with vertex $v_{0}$ and basis the full subgraph with vertices $v_{1}$, $v_{2}, v_{3}$. Set $\mathcal{V}^{\text {op }}=\left\{a_{0}, a_{1}, a_{2}, a_{3}\right\}$, with $a_{i}$ dual to $v_{i}$ for all $i$, and $A_{\bullet}=\Lambda_{\bullet}\left(\Gamma^{\mathrm{op}}\right)$. Then

$$
\begin{aligned}
& A_{1}=\left\langle a_{0}, a_{1}, a_{2}, a_{3}\right\rangle, \\
& A_{2}=\left\langle a_{0} a_{1}, a_{0} a_{2}, a_{0} a_{3}, a_{1} a_{2}, a_{2} a_{3}\right\rangle=A_{1} \wedge\left\langle a_{0}, a_{2}\right\rangle, \\
& A_{3}=\left\langle a_{0} a_{1} a_{2}, a_{0} a_{2} a_{3}\right\rangle, \\
& A_{n}=0 \quad \text { for } n \geq 3 .
\end{aligned}
$$

Set

$$
b=\alpha_{0} a_{0}+\alpha_{1} a_{1}+\alpha_{2} a_{2}+\alpha_{3} a_{3} \neq 0 .
$$

Without loss of generality, we can suppose that either $b=a_{2}$ (if $\operatorname{Ann}(b)_{1}$ has dimension 1 ) or $b=a_{3}$ (if $\operatorname{Ann}(b)_{1}$ has dimension 2) by performing a change of 
basis for $A_{1}$ inducing an automorphism of $A_{\bullet}$. For $I=\mathscr{L}\left(A_{\bullet}\right)$ such that $b \notin I_{1}$, set $J=I:(b)$ and $K=\operatorname{Ann}(b) \subseteq J$. In both cases, one has $K_{3}=J_{3}=A_{3}$, whereas $K_{1}=\langle b\rangle$ and $K_{2}=\left\langle b a_{0}, b a_{1}, b a_{3}\right\rangle$ for $b=a_{2}$, and $K_{1}=\left\langle a_{1}, a_{3}\right\rangle$ and $K_{2}=\left\langle a_{0} a_{1}, a_{0} a_{3}, a_{1} a_{2}, a_{2} a_{3}\right\rangle$ for $b=a_{3}$. Moreover, $J_{1}=K_{1}+I_{1}$. In particular, $K \in \mathscr{L}\left(A_{\bullet}\right)$ in both cases so that if $I=(0)$, then $J=K \in \mathscr{L}\left(A_{\bullet}\right)$.

Suppose now that $I \neq(0)$. Then either $\operatorname{dim}\left(I_{3}\right)=1$ or $\operatorname{dim}\left(I_{3}\right)=2$. In particular, $\operatorname{dim}\left(I_{3}\right)=1$ if, and only if, $I=\left(b^{\prime}\right)$ for some $b^{\prime} \in\left\langle a_{1}, a_{3}\right\rangle$, whereas $\operatorname{dim}\left(I_{3}\right)=2$ if, and only if, either $I=\left(b^{\prime}\right)$ for some $b^{\prime} \notin\left\langle a_{1}, a_{3}\right\rangle$, or $\operatorname{dim}\left(I_{1}\right) \geq 2$. Altogether, one has the following cases.

(a) Suppose $\operatorname{dim}\left(I_{3}\right)=1$. If $b=a_{3}$, we may assume without loss of generality that $b^{\prime}=a_{1}$ so that $J_{2}=K_{2}$. If $b=a_{2}$, then $J_{2}=K_{2}+\left\langle a_{0} b^{\prime}\right\rangle \subseteq\left(J_{1}\right)$.

(b) Suppose $I_{3}=A_{3}$ so that $J_{2}=A_{2}$. If $b=a_{3}$, then one may find

$$
b^{\prime \prime}=\gamma_{0} a_{0}+\gamma_{1} a_{1}+\gamma_{2} a_{2} \in J_{1}=K_{1}+I_{1},
$$

with $\gamma_{i} \in \mathbb{F},\left(\gamma_{0}, \gamma_{2}\right) \neq(0,0)$, so that $a_{0} a_{2} \in\left(b^{\prime \prime}\right)_{2}+K_{2}$. Therefore, we have $A_{2}=K_{2} \oplus\left\langle a_{0} a_{2}\right\rangle \subseteq\left(J_{1}\right)$.

If $b=a_{2}$, then one has two further subcases. If $\operatorname{dim}\left(I_{1}\right)=1$, then one may find

$$
b^{\prime \prime}=a_{0}+\gamma_{1} a_{1}+\gamma_{3} a_{3} \in J_{1}=K_{1}+I_{1},
$$

with $\gamma_{1}, \gamma_{3} \in \mathbb{F}$, as $b^{\prime} \notin\left\langle a_{1}, a_{3}\right\rangle$ and $b=a_{2}, b^{\prime} \in J_{1}$. Thus

$$
A_{2}=K_{2} \oplus\left\langle a_{0} a_{1}, a_{0} a_{3}\right\rangle \subseteq\left(J_{1}\right) .
$$

On the other hand, if $\operatorname{dim}\left(I_{1}\right) \geq 2$ and $J_{1}$ does not contain an element $b^{\prime \prime}$ as above, then necessarily $a_{1}, a_{3} \in J_{1}$; in both cases, $a_{0} a_{1}, a_{0} a_{3} \in\left(J_{1}\right)$ and $A_{2}=K_{2} \oplus\left\langle a_{0} a_{1}, a_{0} a_{3}\right\rangle \subseteq\left(J_{1}\right)$.

In every case, one has $J \in \mathscr{L}\left(A_{\bullet}\right)$.

\section{Pro- $p$ groups}

\subsection{Right-angled Artin pro- $p$ groups}

Fix a prime number $p$, and let $\mathbb{F}_{p}$ be the finite field with $p$ elements. For a pro- $p$ group $\mathscr{G}$, we consider $\mathbb{F}_{p}$ as continuous trivial $\mathscr{G}_{\text {-module. The continuous cochain }}$ cohomology algebra

$$
H^{\bullet}\left(\mathscr{G}, \mathbb{F}_{p}\right)=\bigoplus_{n \geq 0} H^{n}\left(\mathscr{E}, \mathbb{F}_{p}\right)
$$


of $\mathcal{E}$ with coefficients in $\mathbb{F}_{p}$, endowed with the graded-commutative cup-product, is a graded $\mathbb{F}_{p}$-algebra. In particular, one has isomorphisms of $p$-elementary abelian groups

$$
H^{1}\left(\mathscr{E}, \mathbb{F}_{p}\right) \simeq \operatorname{Hom}\left(\mathscr{G} / \Phi(\mathscr{G}), \mathbb{F}_{p}\right),
$$

where $\Phi(\mathscr{E})$ denotes the Frattini subgroup of $\mathscr{E}$, i.e., the closed subgroup generated by all $g^{p}$ and $[g, h]$, with $g, h \in \mathcal{E}$. For the definition and the properties of continuous cochain cohomology of pro- $p$ groups we refer to [19, § I.2-I.4].

Given a graph $\Gamma$, we call the pro- $p$ completion $\mathscr{E}_{\Gamma}$ of the RAAG $G_{\Gamma}$ a pro-p $R A A G$ with associated graph $\Gamma$. Pro- $p$ RAAGs behave pretty much like abstract RAAGs (see, e.g., [14]). In particular, one has the following result by K. Lorensen (cf. [16, Theorem 2.6]).

Theorem 5.1. Let $\Gamma$ be a graph. Then $H^{\bullet}\left(\mathscr{G}_{\Gamma}, \mathbb{F}_{p}\right) \simeq H^{\bullet}\left(G_{\Gamma}, \mathbb{F}_{p}\right)$.

Moreover, one has the "pro- $p$ equivalent" of Lemma 3.2 and of (3.1), namely, if a graph $\Gamma$ decomposes into connected components $\Gamma_{1}, \ldots, \Gamma_{r}$, then the pro- $p$ RAAG $\mathscr{E}_{\Gamma}$ decomposes as free pro- $p$ product $\mathscr{E}_{\Gamma} \simeq \mathscr{E}_{\Gamma_{1}} * \hat{p} \cdots * \hat{p} \mathscr{E}_{\Gamma_{r}}$, where $* \hat{p}$ denotes the free product in the category of pro- $p$ groups (see $[32, \S 9.1]$ ), and the $\mathbb{F}_{p}$-cohomology algebra of $\mathscr{E}_{\Gamma}$ decomposes as direct product of quadratic algebras

$$
H^{\bullet}\left(\mathscr{E}_{\Gamma}, \mathbb{F}_{p}\right) \simeq \Lambda_{\bullet}\left(\Gamma^{\mathrm{op}}\right) \simeq \Lambda_{\bullet}\left(\Gamma_{1}^{\mathrm{op}}\right) \sqcap \cdots \sqcap \Lambda_{\bullet}\left(\Gamma_{r}^{\mathrm{op}}\right) .
$$

Thus, one may extend Theorem 1.1 and Theorem 1.2 to the class of pro- $p$ RAAGs.

Theorem 5.2. Let $\Gamma$ be a graph and $\mathscr{E}_{\Gamma}$ the associated pro-p $R A A G$.

(i) The $\mathbb{F}_{p}$-cohomology algebra $H^{\bullet}\left(\mathscr{G}_{\Gamma}, \mathbb{F}\right)$ is strongly Koszul and $P B W$.

(ii) The $\mathbb{F}_{p}$-cohomology algebra $H^{\bullet}\left(\mathcal{E}_{\Gamma}, \mathbb{F}\right)$ is universally Koszul if, and only if, $\Gamma$ has the diagonal property.

A class of pro- $p$ groups which are very similar to pro- $p$ RAAGs is the class of generalized pro- $p$ RAAGs, which was introduced and studied in [31]. Given a graph $\Gamma=(\mathcal{V}, \mathcal{E})$, a generalized pro- $p$ RAAG with associated graph $\Gamma$ is a pro- $p$ group $\mathcal{G}$ generated by $\mathcal{V}=\left\{v_{1}, \ldots, v_{d}\right\}$ and with defining relations $\left[v_{i}, v_{j}\right] v_{i}^{\alpha} v_{i}^{\beta}$ for some $\alpha, \beta \in p \mathbb{Z}_{p}$ if $\left(v_{i}, v_{j}\right) \in \mathcal{E}$ (moreover, $\alpha, \beta \in 4 \mathbb{Z}_{2}$ if $p=2$ ). Namely, one has a presentation (of pro- $p$ groups)

$$
\mathcal{E}=\left\langle v_{1}, \ldots, v_{d} \mid\left[v_{i}, v_{j}\right] v_{i}^{\alpha_{i j}} v_{i}^{\beta_{i j}}=1,\left(v_{i}, v_{j}\right) \in \mathcal{E}, \alpha_{i j}, \beta_{i j} \in p^{\epsilon} \mathbb{Z}_{p}\right\rangle_{\hat{p}},
$$

with $\epsilon=1$ if $p>2, \epsilon=2$ otherwise.

A priori, a generalized pro- $p$ RAAG may have $\mathbb{F}_{p}$-cohomology algebra which is not quadratic - e.g., a generalized pro- $p$ RAAG $\mathscr{E}$ may be a finite group so that 
$H^{\bullet}\left(\mathscr{G}, \mathbb{F}_{p}\right)$ is not quadratic unless $p=2$ and $\mathscr{Y}$ is 2-elementary abelian (cf. [31, Example 5.16]). Yet, if $H^{\bullet}\left(\mathscr{G}, \mathbb{F}_{p}\right)$ is quadratic for a generalized pro- $p$ RAAG $\mathscr{G}$ with associated graph $\Gamma$, then one has $H^{\bullet}\left(\mathscr{G}, \mathbb{F}_{p}\right) \simeq \Lambda_{\bullet}\left(\Gamma^{\mathrm{op}}\right)$, just like (pro- $p$ ) RAAGs (cf. [31, Theorem E]). Hence, by Theorem 4.3, the $\mathbb{F}_{p}$-cohomology algebra of a generalized pro- $p$ RAAG $\mathcal{E}$ is strongly Koszul, too. In $[31, \S 5.5,5.6]$, uncountably many examples of generalized pro- $p$ RAAGs are shown to have quadratic $\mathbb{F}_{p}$-cohomology algebra - e.g., if $\Gamma$ contains no triangles as full subgraphs (cf. [31, Theorem F]). Thus, one may deduce the following.

Corollary 5.3. Let $\mathcal{E}$ be a generalized pro- $p$ RAAG with associated graph $\Gamma$, and suppose that $H^{\bullet}\left(\mathcal{G}, \mathbb{F}_{p}\right)$ is a quadratic algebra. Then $H^{\bullet}\left(\mathscr{G}, \mathbb{F}_{p}\right)$ is a strongly Koszul and PBW algebra. Moreover, it is universally Koszul if, and only if, $\Gamma$ has the diagonal property.

Example 5.4. Let $\mathcal{G}$ be the pro- $p$ group with presentation

$$
\mathcal{E} \simeq\left\langle w, v_{1}, \ldots, v_{d} \mid\left[v_{i}, w\right]=v_{i}^{\alpha_{i}}, i=1, \ldots, d, \alpha_{i} \in p^{\epsilon} \mathbb{Z}_{p}\right\rangle_{\hat{p}},
$$

where $\epsilon=1$ if $p>2, \epsilon=2$ otherwise. Then $\mathscr{E}$ is a generalized pro- $p$ RAAG with associated graph a star graph $\Gamma$ with center $w$. Thus, by [31, Theorem F], one has $H^{\bullet}\left(\mathscr{g}, \mathbb{F}_{p}\right) \simeq \Lambda_{\bullet}\left(\Gamma^{\mathrm{op}}\right)$, which is strongly and universally Koszul, and PBW.

Example 5.5. Let $\mathscr{E}$ be the pro- $p$ group with presentation

$$
\mathcal{E} \simeq\left\langle v_{0}, \ldots, v_{4} \mid\left[v_{0}, v_{i}\right]=v_{0}^{\alpha_{i}} v_{i}^{\beta_{i}},\left[v_{1}, v_{2}\right]=v_{1}^{\alpha_{1}^{\prime}} v_{2}^{\beta_{1}^{\prime}},\left[v_{2}, v_{3}\right]=v_{2}^{\alpha_{3}^{\prime}} v_{3}^{\beta_{3}^{\prime}}\right\rangle_{\hat{p}},
$$

with $i=1, \ldots, 4$, and $\alpha_{i}, \beta_{i}, \alpha_{i}^{\prime}, \beta_{i}^{\prime} \in p^{\epsilon} \mathbb{Z}_{p}$, where $\epsilon=1$ if $p>2, \epsilon=2$ otherwise. Then $\mathscr{Y}$ is a generalized pro- $p$ RAAG with associated graph

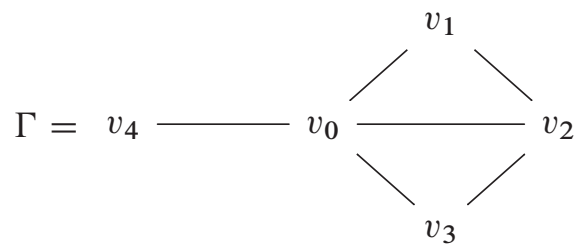

The graph $\Gamma$ is the cone graph with basis the full subgraph with vertices $v_{1}, \ldots, v_{4}$, and it has the diagonal property. By $[31, \S 5.6]$, one has $H^{\bullet}\left(\mathscr{g}, \mathbb{F}_{p}\right) \simeq \Lambda_{\bullet}\left(\Gamma^{\mathrm{op}}\right)$, which is strongly and universally Koszul, and PBW.

Example 5.6. Let $\mathscr{E}$ be the pro- $p$ group with presentation

$$
\mathcal{E} \simeq\left\langle v_{0}, \ldots, v_{4} \mid\left[v_{0}, v_{i}\right]=v_{0}^{\alpha_{i}} v_{i}^{\alpha_{i}},\left[v_{1}, v_{j}\right]=v_{1}^{\alpha^{\prime}} v_{j}^{\beta^{\prime}},\left[v_{4}, v_{j}\right]=v_{4}^{\alpha^{\prime \prime}} v_{j}^{\beta^{\prime \prime}}\right\rangle_{\hat{p}},
$$


with $i=1,4, j=2,3$, and $\alpha_{i}, \beta_{i}, \alpha^{\prime}, \beta^{\prime}, \alpha^{\prime \prime}, \beta^{\prime \prime} \in p^{\epsilon} \mathbb{Z}_{p}$, where $\epsilon=1$ if $p>2$, $\epsilon=2$ otherwise. Then $\mathscr{G}$ is a generalized pro- $p$ RAAG with associated graph

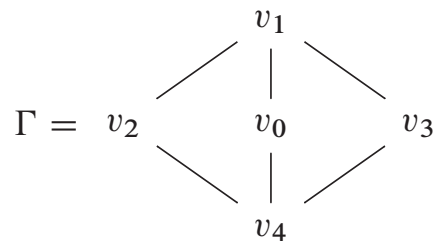

The graph $\Gamma$ does not have the diagonal property. By $[31, \S 5.6]$, one has

$$
H^{\bullet}\left(\mathscr{G}, \mathbb{F}_{p}\right) \simeq \Lambda_{\bullet}\left(\Gamma^{\mathrm{op}}\right),
$$

which is strongly Koszul and PBW, but not universally Koszul.

One may find a further class of pro- $p$ groups whose $\mathbb{F}_{p}$-cohomology algebras are both strongly and universal Koszul. A finitely generated pro- $p$ group is called uniform if it is torsion-free and $\Phi(\mathscr{E}) \subseteq \overline{\mathscr{E}}^{p^{\epsilon}}$, where $\overline{\mathscr{E}} p^{\epsilon}$ denotes the closed subgroup of $\mathcal{E}$ generated by the $p^{\epsilon}$-powers of elements of $\mathcal{E}$, where $\epsilon=1$ if $p>2$ and $\epsilon=2$ otherwise (cf. [7, §4.1]). For these pro- $p$ groups one has the following.

Proposition 5.7. Let $\mathcal{E}$ be a uniform pro-p group. Then the $\mathbb{F}_{p}$-cohomology algebra $H^{\bullet}\left(\mathcal{G}, \mathbb{F}_{p}\right)$ is strongly and universally Koszul, and PBW.

Proof. Set $V=H^{1}\left(\mathcal{G}, \mathbb{F}_{p}\right)$. Then, by [35, Theorem 5.1.5], one has

$$
H^{\bullet}\left(\mathscr{G}, \mathbb{F}_{p}\right) \simeq \Lambda_{\bullet}(V) .
$$

The claim follows from Theorem 4.3 and Theorem 4.6.

\subsection{Maximal pro- $p$ Galois groups}

In this subsection, $\mathbb{K}$ will denote a field containing a root of 1 of order $p$. By the positive answer to the Bloch-Kato conjecture given by M. Rost and V. Voevodsky (cf. $[33,36,37]$ ), one knows that the maximal pro- $p$ Galois group $\mathscr{E}_{\mathbb{K}}$ of $\mathbb{K}$ has quadratic $\mathbb{F}_{p}$-cohomology algebra (see, e.g., $[28, \S 2]$ ). In [17], it is conjectured that $\mathscr{E}_{\mathbb{K}}$ has universally Koszul $\mathbb{F}_{p}$-cohomology algebra (cf. Conjecture 1.3).

Proposition 5.8. If $\Gamma$ is a graph with the diagonal property, then, for any prime $p$, the pro-p $R A A G \mathscr{E}_{\Gamma}$ associated to $\Gamma$ occurs as the maximal pro-p Galois group $\mathscr{E}_{\mathbb{K}}$ for some field $\mathbb{K}$ containing a root of 1 of order $p$. 
Proof. If a finitely generated pro- $p$ group $\mathcal{E}$ occurs as the maximal pro- $p$ Galois group $\mathscr{E}_{\mathbb{K}}$ for some field $\mathbb{K}$ containing a root of 1 of order $p$, then one has

$$
\mathcal{G} \times \mathbb{Z}_{p} \simeq \mathscr{E}_{\mathbb{K}((X))},
$$

with $\mathbb{K}((X))$ the field of Laurent series in one indeterminate $X$ with coefficients in $\mathbb{K}$. In particular,

$$
\mathbb{Z}_{p}=\{1\} \times \mathbb{Z}_{p} \simeq \mathscr{E}_{\mathbb{K}((X))},
$$

with $\mathbb{K}$ a $p$-closed field (i.e., $\mathscr{E}_{\mathbb{K}}=\{1\}$ ). On the other hand, if two finitely generated pro- $p$ groups $\mathscr{E}_{1}, \mathscr{E}_{2}$ occur as maximal pro- $p$ Galois groups, then also their free product is realizable as the maximal pro- $p$ Galois group for some field $\mathbb{K}$ (cf. [9, Remark 3.4]).

We proceed by induction on the number $d$ of vertices of $\Gamma$. If $\Gamma$ has the diagonal property, then $\varepsilon_{\Gamma}$ is constructible starting from free abelian pro- $p$ groups by taking direct product with $\mathbb{Z}_{p}$ and free products. Therefore, if $d=1$, then $\mathscr{E}_{\Gamma} \simeq \mathbb{Z} \simeq \mathscr{E}_{\mathbb{K}}$ for some suitable $\mathbb{K}$. If $d \geq 2$, then either $\mathscr{S}_{\Gamma} \simeq \mathscr{E}_{\Gamma_{1}} * \hat{p} \mathscr{E}_{\Gamma_{2}}$, with $\Gamma=\Gamma_{1} \dot{\cup} \Gamma_{2}$, or $\mathscr{E}_{\Gamma} \simeq \mathbb{Z} \times \mathscr{E}_{\tilde{\Gamma}}$, with $\Gamma$ the cone graph with basis $\tilde{\Gamma}$, and the claim follows by the above argument.

Remark 5.9. If $\Gamma$ is a graph with the diagonal property, then the associated pro- $p$ RAAG $e_{\Gamma}$ is a pro- $p$ group of elementary type (cf. $[9, \S 3]$ and $[18, \S 4]$ ).

On the other hand, it was recently shown that the only pro- $p$ RAAGs which may occur as maximal pro- $p$ Galois groups of fields containing a root of 1 of order $p$ are those associated to graphs with the diagonal property. Indeed, let $C_{4}$ and $P_{4}$ be as in (3.2). The pro- $p$ RAAG $\mathscr{G}_{C_{4}}$ associated to $C_{4}$ is isomorphic to the direct product $\mathscr{F} \times \mathcal{F}$, with $\mathscr{F}$ a 2 -generated free pro- $p$ group, and by [28, Theorem 5.6], $\mathscr{G}_{C_{4}}$ - and therefore also any other pro- $p$ RAAG $\mathscr{E}_{\Gamma}$ with $\Gamma$ containing $C_{4}$ as full subgraph - is not realizable as the maximal pro- $p$ Galois group $\mathscr{E}_{\mathbb{K}}$ for any $\mathbb{K}$. Moreover, I. Snopce and P. Zalesskii recently proved that ${\boldsymbol{P _ { P }}}_{P_{4}}$ and therefore also any other pro- $p$ RAAG $\mathscr{E}_{\Gamma}$ with $\Gamma$ containing $P_{4}$ as full subgraph - is not realizable as the maximal pro- $p$ Galois group $\mathscr{E}_{\mathbb{K}}$ for any $\mathbb{K}$ (cf. [34, Theorem 1.2]).

From this, together with Proposition 5.8 and Theorem 5.2, one deduces Corollary 1.3.

Remark 5.10. Unlike pro- $p$ RAAGs, not every generalized pro- $p$ RAAG with associated graph a graph with the diagonal property occurs as the maximal pro- $p$ Galois group $\mathscr{E}_{\mathbb{K}}$ of a field $\mathbb{K}$ containing a root of 1 of order $p$, as shown by the following examples. 
(a) Let $\mathcal{G}$ be as in Example 5.4. If $\alpha_{i} \neq \alpha_{j}$ for some $i, j$, then $\mathcal{Z} \not \mathscr{E}_{\mathbb{K}}$ for any field $\mathbb{K}$ containing a root of 1 of order $p$ by [10, Example 8.3].

(b) Let $\mathscr{E}$ be as in Example 5.5. If $\alpha_{1}=\beta_{4}=p$ and $\beta_{1}=\alpha_{4}=0$, then $\mathscr{\mathcal { G }} \not \mathscr{E}_{\mathbb{K}}$ for any field $\mathbb{K}$ containing a root of 1 of order $p$ by [31, Theorem 5.29].

Acknowledgments. The authors are grateful to F. W. Pasini, as this paper was inspired by the talk he delivered at the "Mathematical Salad" seminars at the University of Milano-Bicocca, Italy, in Dec. 2018, and to the organizers of that talk. Also, the authors wish to thank P. Spiga for the discussions with him about graphs, S. Blumer and F. Matucci for their comments, and I. Snopce and M. Vannacci, as the joint work with the second-named author on pro- $p$ RAAGs was also source of inspiration for this paper.

\section{Bibliography}

[1] R. Charney, An introduction to right-angled Artin groups, Geom. Dedicata 125 (2007), 141-158.

[2] A. Conca, Gröbner bases for spaces of quadrics of low codimension, Adv. Appl. Math. 24 (2000), no. 2, 111-124.

[3] A. Conca, Universally Koszul algebras, Math. Ann. 317 (2000), no. 2, 329-346.

[4] A. Conca, E. De Negri and M. E. Rossi, Koszul algebras and regularity, in: Commutative Algebra, Springer, New York (2013), 285-315.

[5] A. Conca, N. V. Trung and G. Valla, Koszul property for points in projective spaces, Math. Scand. 89 (2001), no. 2, 201-216.

[6] R. Diestel, Graph Theory, 5th ed., Grad. Texts in Math. 173, Springer, Berlin, 2018.

[7] J.D. Dixon, M.P.F. du Sautoy, A. Mann and D. Segal, Analytic pro-p Groups, 2nd ed., Cambridge Stud. Adv. Math. 61, Cambridge University, Cambridge, 1999.

[8] C. Droms, Subgroups of graph groups, J. Algebra 110 (1987), no. 2, 519-522.

[9] I. Efrat, Small maximal pro- $p$ Galois groups, Manuscripta Math. 95 (1998), no. 2, 237-249.

[10] I. Efrat and C. Quadrelli, The Kummerian property and maximal pro- $p$ Galois groups, J. Algebra 525 (2019), 284-310.

[11] V. Ene, J. Herzog and T. Hibi, Linear flags and Koszul filtrations, Kyoto J. Math. 55 (2015), no. 3, 517-530.

[12] R. Fröberg, Determination of a class of Poincaré series, Math. Scand. 37 (1975), no. $1,29-39$. 
[13] J. Herzog, T. Hibi and G. Restuccia, Strongly Koszul algebras, Math. Scand. 86 (2000), no. 2, 161-178.

[14] R. Kropholler and G. Wilkes, Profinite properties of RAAGs and special groups, Bull. Lond. Math. Soc. 48 (2016), no. 6, 1001-1007.

[15] J.-L. Loday and B. Vallette, Algebraic Operads, Grundlehren Math. Wiss. 346, Springer, Heidelberg, 2012.

[16] K. Lorensen, Groups with the same cohomology as their pro- $p$ completions, J. Pure Appl. Algebra 214 (2010), no. 1, 6-14.

[17] J. Mináč, M. Palaisti, F. W. Pasini and N. D. Tân, Enhanced Koszul properties in Galois cohomology, Res. Math. Sci. 7 (2020), no. 2, Paper No. 10.

[18] J. Mináč, F. W. Pasini, C. Quadrelli and N. D. Tân, Koszul algebras and quadratic duals in galois cohomology, preprint (2018), https://arxiv.org/abs/1808. 01695.

[19] J. Neukirch, A. Schmidt and K. Wingberg, Cohomology of Number Fields, 2nd ed., Grundlehren Math. Wiss. 323, Springer, Berlin, 2008.

[20] S. Papadima and A. I. Suciu, Algebraic invariants for right-angled Artin groups, Math. Ann. 334 (2006), no. 3, 533-555.

[21] D. I. Piontkovskiŭ, Koszul algebras and their ideals, Funktsional. Anal. i Prilozhen. 39 (2005), no. 2, 47-60, 95.

[22] A. Polishchuk and L. Positselski, Quadratic Algebras, Univ. Lecture Ser. 37, American Mathematical Society, Providence, 2005.

[23] L. Positselski, Koszul property and Bogomolov's conjecture, Int. Math. Res. Not. IMRN 2005 (2005), no. 31, 1901-1936.

[24] L. Positselski, Galois cohomology of a number field is Koszul, J. Number Theory 145 (2014), 126-152.

[25] L. Positselski, Koszulity of cohomology $=K(\pi, 1)$-ness + quasi-formality, J. Algebra 483 (2017), 188-229.

[26] L. Positselski and A. Vishik, Koszul duality and Galois cohomology, Math. Res. Lett. 2 (1995), no. 6, 771-781.

[27] S. B. Priddy, Koszul resolutions, Trans. Amer. Math. Soc. 152 (1970), 39-60.

[28] C. Quadrelli, Bloch-Kato pro- $p$ groups and locally powerful groups, Forum Math. 26 (2014), no. 3, 793-814.

[29] C. Quadrelli, One-relator maximal pro- $p$ galois groups and the koszulity conjectures, preprint (2016), https://arxiv.org/abs/1601.04480; to appear in Q. J. Math.

[30] C. Quadrelli, Pro- $p$ groups with few relations and universal koszulity, preprint (2020), https: //arxiv . org/abs/2003.09186; to appear in Math. Scand. 
[31] C. Quadrelli, I. Snopce and M. Vannacci, On pro- $p$ groups with quadratic cohomology, preprint (2019), https: //arxiv org/abs/1906.04789.

[32] L. Ribes and P. Zalesskii, Profinite Groups, 2nd ed., Ergeb. Math. Grenzgeb. (3) 40, Springer, Berlin, 2010.

[33] M. Rost, Norm varieties and algebraic cobordism, in: Proceedings of the International Congress of Mathematicians. Vol. II (Beijing 2002), Higher Education, Beijing (2002), 77-85.

[34] I. Snopce and P. A. Zalesskii, Right-angled artin pro- $p$ groups, preprint (2020), https://arxiv.org/abs/2005.01685.

[35] P. Symonds and T. Weigel, Cohomology of $p$-adic analytic groups, in: New Horizons in pro-p Groups, Progr. Math. 184, Birkhäuser, Boston (2000), 349-410.

[36] V. Voevodsky, On motivic cohomology with Z/l-coefficients, Ann. of Math. (2) 174 (2011), no. 1, 401-438.

[37] C. Weibel, 2007 Trieste lectures on the proof of the Bloch-Kato conjecture, in: Some Recent Developments in Algebraic K-theory, ICTP Lect. Notes 23, Abdus Salam Int. Cent. Theoret. Phys., Trieste (2008), 277-305.

[38] T. Weigel, Graded Lie algebras of type FP, Israel J. Math. 205 (2015), no. 1, 185209.

[39] E. S. Wolk, A note on "The comparability graph of a tree", Proc. Amer. Math. Soc. 16 (1965), 17-20.

Received April 2, 2020; revised July 10, 2020.

\section{Author information}

Alberto Cassella, Department of Mathematics and Applications,

University of Milano Bicocca, 20125 Milan, Italy.

E-mail: a.cassella@campus .unimib.it

Corresponding author:

Claudio Quadrelli, Department of Mathematics and Applications,

University of Milano Bicocca, 20125 Milan, Italy.

E-mail: claudio.quadrelli@unimib.it 